\title{
Analysis of the Characteristics and Types of Network Program Hosts
}

\author{
Lei $\mathrm{Xi}^{\text {a }}$, Zaining Wang ${ }^{\mathrm{b}, *}$ \\ School of Journalism and Communication, China West Normal University, Nanchong, 637009 Sichuan, \\ China \\ a227468855@qq.com, b58993597@qq.com \\ *Corresponding author
}

Keywords: traditional media, network programming, features, genres.

\begin{abstract}
The rapid development of the Internet has brought innovations in all areas of the media industry, and traditional media are facing increasing challenges from emerging media. Online video programs have attracted a large number of young people due to their broader topics and free production space. The debut of new media allowed the new concept of web host to emerge. Due to the inherent characteristics of online program dissemination, network hosts have lower entry barriers than traditional media hosts, and also have more distinctive personal characteristics. The innovation brought about by the new media platform in the moderator industry brings not only opportunities, but also challenges and risks. By sorting out the development trends of network video program hosts in recent years, we can find that there are qualitative to quantitative changes in characteristics, types, and development trends.
\end{abstract}

\section{Introduction}

Communication expert McLuhan believes that the emerging media not only creates new ways of social life and cultural information dissemination, but also in a sense is the driving force for social development. The data shows that in the past few years, the number and influence of online video have shown a geometric upward trend. In the new media platform, online video and programs have shown an upward trend in the scope of influence and the number of people affected. In addition to that the large student population is the largest audience characteristic of online platform programs, the age level, gender distribution, and income level of the audience are increasingly showing the characteristics of transition to the middle class of society. This means that the influence of new media is more and more recognized by mainstream values, and the video programs of online platforms are more and more of the nature of mass media. In the face of such a huge audience, the host of the video and program as an online platform becomes more and more important. How to distinguish the host from traditional media in terms of hosting style and skills, how to set the corresponding host positioning for different network programs, and what foreseeable changes in the future have become an important way of thinking to study this particular group of network hosts .

\section{Characteristics of network program host}

\subsection{The network host has no geographical restrictions}

Because the coverage of traditional media TV signals is different, and the coverage is often limited by the spread of the broadcast, the host of the traditional media has the characteristics of being limited by the spread of the broadcast. The host of the local TV station can only be understood by the regional audience covered by the signal, and is often referred to as "Hunan TV program host", "Xinjiang TV program host" or other names with regional characteristics. The corresponding network program host does not have such a problem. The audience of the network is all netizens. Without the geographical restrictions, it also eliminates the narrow field of view brought by the local culture, and has the generalized attributes of the mass host. At the same time, it also puts forward 
higher requirements on the cultural level of the web host. In the face of audiences in different regions, they must be able to be treated equally and face different regional cultures.

\subsection{Network moderators are often non-technical students}

Because the broadcast cycle of network programs is not as fixed as the programs of traditional media, most of the network program hosts come by guest stars or content creators. Online programming has a more liberal creative concept, and the cost is inferior to traditional media. The choice of the host is mainly influenced by non-full-time factors such as suitable style and working hours. Web hosts often come from other professions in their lives, or are experts in the fields involved in the show. For example, the host of "Bao Zou Da Shi Jian" Wang Nima is a science graduate. Out of interest in online culture and personal hobbies, he created the "Bao Zou Da Shi Jian" language talk show program, and the host of the program is also himself. It perfectly reflects the integrated relationship between the main creator and the host. The "Xiao Shuo" produced by Youku is hosted by star singer Gao Xiaosong. His sharp words and erudition fully show the level of hosting that non-professional hosts can achieve in online programs.

\subsection{Two-way function of hosting and interaction}

Live webcasts use the two-way immediacy of online communication to set up a link that interacts with the audience at the same time as content is disseminated. In the traditional media in the past, there have also been two-way communication forms such as SMS interaction, but most of the technical obstacles and operational difficulties are only superficial. It is difficult to achieve the true meaning of the communicator and the audience at the same time. Interactive. In many of today's Internet programs, there will be a chat area on the live web page. While listening to the program, they will express their thoughts and feelings. The host sees these feedbacks at the first time, can communicate quickly, and the listeners who listen to the program can also see each other's message feedback.

\subsection{Extensive use of online buzzwords}

Since the birth of the Internet, its fresh ability to look at things and perspective is its magic weapon. The network vocabulary with original power dissolves the authority of traditional culture, and brings a lively cultural interest and a popular spirit of entertainment. This is a concrete manifestation of the grassroots attributes of Internet culture, and is also the source of the endless vitality of Internet culture. Unlike the ideological functions and tasks of traditional media, network program hosts can use the current online hot words to analyze events and express opinions more freely in the use of language. For example, "beating soy sauce" refers to being indifferent to public topics, and the word "jiong" is used to express facial expressions when depressed, sad, and helpless. The vitality brought about by this new language can make online programs more quickly accepted by the public, and can also be more clearly separated from the serious traditional media programs.

\subsection{The use of virtual characters by web hosting}

The first virtual presenter on the Internet was named Ananova, a computer-simulated newscaster launched by the British Press New Media Company in 1999. The first TV virtual host in China was launched by Beijing TV station "Science and Technology News Weekly" in January 2001. It is an animated character named "Bill Deng". The rapid development of digital technology is jaw-dropping. Nowadays, whether it is in the production of online media or traditional media, the construction of virtual characters or even virtual scenes is no longer a rare event. It is no longer a unique feature of online media that a virtual character becomes a program host. It can be seen that technology exists to serve content forms. As long as it is beneficial to the production of the program, then the traditional media will also cast aside to learn from the new media video program. 


\section{The Classification of Network Host}

At present, there are various types of network programs and endless patterns, showing the creator's flexible imagination after breaking away from the constraints of traditional media program production. However, there is no systematic classification of the current network programs, so the network host also lacks a systematic and logically integrated study. The author here attempts to make a brief classification standard for the identity characteristics of its presenters through most of the network programs that have appeared.

\subsection{Network program host based on traditional media.}

The expansion of traditional TV media in the field of new media has caused some new media platforms to actually depend on the power of traditional media. On this type of website platform, traditional media TV programs are directly transferred to the network, or network video programs with relevant meanings for the corresponding settings of traditional media programs. The host of these programs is also a host of traditional media, also known as the network host. On the one hand, this kind of program content is supported by the traditional media production team, which ensures the excellence of the program quality, and further integrates the communication advantages of the network platform. The hosts also have good professional qualities. The identity of the host with the pure network host.

\subsection{Network program host based on portal website and video website.}

With the increasing influence of online video, various video websites and portal websites have created self-made programs or Internet TV stations suitable for their own positioning. For example, "Sina TV" is a network TV station run by Sina.com, and its original channel is a platform for Sina.com to broadcast its own network programs. The original programs include entertainment programs, financial programs, sports programs and cultural programs. The program type and content are relatively standard, but the broadcast time of the program is uncertain, and a news announcement will be published on the website in advance before the broadcast. LeTV, Sohu and other websites have invested huge amounts of money and manpower on the website's self-made programs, and have achieved good results so far. Some of the hosts of this kind of network programs are employees with certain professional qualities hired by the website itself, some are star commentators or guests, and some are even network celebrities and topical characters. The common feature of such network program hosts is their complicated identity, and most of them do not have professional host qualifications. However, from the perspective of hosting effects, because they are very suitable for the form, style, and professionalism of network programs, such hosts are often more suitable for the control and interpretation of such network video programs than those of professional courses.

\section{The web host who appeared on the forum and the theme website}

The forum is a virtual community with common characteristics for group establishment and activities. It provides a platform for communication and gathering for people with certain interests. The network programs generated on the forums and theme websites are relatively active, usually in the form of live broadcasts, focusing on real-time communication and interactive communication with netizens, and even through the "hotline" direct communication to achieve the effect of network transmission and live broadcast atmosphere. The host who hosts this type of network program represents a forum or a website to a certain extent, so it must have basic broadcaster host requirements, and at the same time-must have a certain degree of understanding of this forum or website.

Most of the hosts of the forums and websites are themselves resident netizens of the forums and websites. They have a certain degree of understanding of the operation and topics of the entire website. These programs are highly targeted and very clear. Audience. At the same time, such network programs are largely borne by the host's own collection, editing, and broadcasting functions, and the program has great flexibility. However, due to the great freedom of this type of network, the 
roles and functions of network program hosts in it are too diverse, which has certain requirements on the host' s professional qualities and comprehensive capabilities, resulting in such network program hosts. The level of people is also uneven.

\section{About the future development of network program hosts}

The dissemination characteristics of the Internet make the presenters of the Internet have a congenital defect. The great charm of the Internet is that everyone becomes part of the message, either as a sharer or as a receiver. This characteristic with the nature of acquisition and participation determines that the level of network program hosts may be uneven. The free nature of network resources lowers the threshold for the production of network programs, and mean that web hosting can be regarded as a sideline or hobby, and the quality of network programs is uneven. Therefore, the production mode of online video programs will still be a road with rising space. The quality of online programs is necessary to be greatly improved. The host of online programs will also improve their business levels,Hosting professional skills and unique hosting styles as the level of program production improves. The network program host can develop towards a high-quality, high-level, and more professional direction, which will greatly help the standardization of the network program host's quality, network program content, and broadcast form.

\section{References}

[1] Song Xinmin. Program Hosting in the Internet Era [J]. Huazhong University of Science and Technology Press, 2006.

[2] Ming Anxiang. Information highway and mass communication [J]. China Publishing House, 1999.

[3] Zhang Yaoxue, Zhang Chi. Entering the Internet World [J]. Tsinghua University Press, 2000.

[4] Wang Xiaowei. Practical Internet Popular Terms [J]. Chinese Dictionary Press. 